ORIGINAL ARTICLE

\title{
Retrospective evaluation of antimicrobial prophylaxis in prevention of surgical site infection in the pediatric population
}

\author{
Goonjan S. Shah ${ }^{1}$, Robert E. Christensen ${ }^{2}$, Deborah S. Wagner ${ }^{2}$, Bridget K. Pearce ${ }^{2}$, \\ Jennifer Sweeney ${ }^{3}$ \& Alan R. Tait ${ }^{2}$ \\ 1 Department of Anesthesiology, University of North Carolina, Chapel Hill, NC, USA \\ 2 Department of Anesthesiology, University of Michigan Health System, Ann Arbor, MI, USA \\ 3 Department Infection Control \& Epidemiology, University of Michigan Health System, Ann Arbor, MI, USA
}

\author{
Keywords \\ surgical wound infection; postoperative \\ complications; antibiotic prophylaxis; \\ antibacterial agents; risk factors; child

\section{Correspondence \\ Dr. Goonjan S. Shah, Department of Anesthesia, University of North Carolina, 2100 Old Dental Building, CB\#7455, Chapel Hill, NC 27599-7455, USA \\ Email: gshah@aims.unc.edu}

Section Editor: Andrew Davidson

Accepted 13 April 2014

doi:10.1111/pan.12436

\begin{abstract}
Summary
Background and Objectives: Few studies have been conducted in pediatric patients evaluating efficacy of prophylactic antibiotics for prevention of surgical site infection (SSI). This retrospective study was undertaken to determine the effect of antibiotic prophylaxis in the prevention of SSI in children.

Methods: With IRB approval, our perioperative electronic clinical information database was queried. Pediatric patients ( $\leq 18$ years) undergoing general surgery, cardiac surgery, and spinal surgery at Mott Children's Hospital from January 2000 to April 2010 were included. Demographics and preoperative data were obtained from the Centricity Intraoperative Database, and any episodes of SSI were obtained by review of the infection control records.

Results: A total 5023 pediatric patients underwent surgery from January 2000 to April 2010. The average age of the children in the sample was $4.16 \pm 5.5$ years, and of these, $57 \%$ were boys. Overall, 119 (2.37\%) cases of SSI were identified. There were no associations between the various patient factors and the development of SSIs. Children for whom antibiotics were administered incorrectly had a 1.7-fold increased risk of SSIs compared with children who received antibiotics within the recommended guidelines $(P<0.02)$. Children who received antibiotics were more likely to suffer an SSI compared with those who did not.

Conclusions: Proper administration of preoperative antibiotics in pediatric patients is one of the few modifiable and significant factors in prevention of SSI.
\end{abstract}

\section{Introduction}

Surgical site infection (SSI) accounts for $14-16 \%$ of nosocomial infections in the United States (1). SSI adds greatly to the cost of hospitalization and increases morbidity and mortality (2). In the pediatric population, it has been shown to increase hospital stay by 10.6 days and increase the cost of hospitalization by $\$ 27000$ (3). The incidence of SSIs in the pediatric population ranges from $2.5 \%$ to $20 \%$ (4-10). Current guidelines define appropriate antibiotic prophylaxis for SSI as administration within $60 \mathrm{~min}$ prior to incision
(11). Although antibiotic prophylaxis given within $1 \mathrm{~h}$ of surgical incision has been shown to be effective in preventing surgical infections in breast, appendix, and colorectal surgery in adults (2), there have been few studies regarding the efficacy of this prophylactic guideline for antibiotics administration for prevention of SSI in children. Currently, prophylactic antibiotic guidelines exist for certain subsets of the pediatric surgical population, but there are no global recommendations, and the guidelines that exist are mostly based on studies from adults or from expert opinion $(1,11,12)$. 
In one study, Horwitz et al. examined risk factors for wound infection in children and found that preoperative antibiotics did not influence the development of SSI. However, there was no attempt to standardize the types of antibiotics used or the overall duration of therapy, making it difficult to make conclusions regarding the efficacy of prophylactic antibiotics on SSI reduction (8). Another study conducted by Porras-Hernandez et al. examined risk factors for SSI in a pediatric hospital and found that antibiotic prophylaxis was marginally significant as a protective factor. However, the power of this study was low and multivariate analysis was not performed, and furthermore, there was an unusually high baseline infection rate of $18.7 \%$ (13). In another study, Bucher et al. (14) examined risk factors and outcomes in children who developed SSI and determined that antibiotic administration was not an independent predictor of SSI. Most recently, Hawn et al. evaluated the timing of antibiotic prophylaxis using data from the National Veterans Affairs Database and concluded that SSI risk factors were associated with patient and procedure factors, but not with antibiotic prophylaxis timing (15).

To date, no study has specifically sought to answer the direct impact of prophylactic antibiotics on SSI development in children. This retrospective study therefore was undertaken to evaluate whether appropriate antibiotic prophylaxis, within 60 min prior to incision and appropriate dosage, is associated with a reduction in SSIs in children undergoing a variety of surgical procedures.

\section{Materials and methods}

With institutional review board approval, the perioperative electronic clinical information database (Centricity ${ }^{\circledR}$ General Electric Healthcare, Waukesha, Wisconsin) was queried to identify pediatric patients who underwent surgery at CS Mott Children's Hospital from July 2008 to April 2010. All pediatric patients (age 18 or younger) undergoing general surgery, cardiac surgery, or spinal surgery were included. Additionally, cardiac surgery cases from January 2000 to April 2010 and spinal surgery cases from January 2006 to April 2010 were included. Patient demographics and preoperative data including age, gender, surgical procedure, and presence of comorbidities (i.e., upper respiratory tract infections, malignancy, and diabetes) were obtained from the Centricity Perioperative Database. Details regarding the administration of antibiotics were also recorded. Failure to administer antibiotics correctly was defined as administration greater than $1 \mathrm{~h}$ prior to surgical incision, after surgical incision, or administration of a dose below the recommended weight per kilogram guidelines as defined by the University of Michigan's antibiotic prophylaxis guidelines for pediatric patients. At our institution, Infection Control and Epidemiology follows all cases after surgery for at least 30 days to monitor for development of SSI and documents these. These records are extracted from the examined.

Infection Control and Epidemiology staff evaluated all patients for SSIs who underwent surgery at CS Mott Children's Hospital operating rooms per protocol set by the Infection Control and Epidemiology Department. All SSI were determined using CDC/NHSN definitions. The cases of SSI development were monitored, and data were collected. Additionally, medical records of patients who underwent surgery were queried to identify any SSI that may not have been cultured for organisms. The keywords used to identify possible SSI included: redness, red, surgical site infection, wound infection, wound infected, wound vacuum assisted closure, wound packing, wound packed, open wound, incision, deep, superficial, debride, dehisce, osteomyelitis, mastitis, endometritis, endocarditis, mediastinitis, meningitis, perforation, ventriculitis, leak, sinusitis, cellulitis, fascia, eviscer purulent, purulence, draining, drainage, abscess, culture, erythema, tender, swelling, swollen, infect, stitch, warm, fever, hematoma, itis, pus, seroma, yellow, brown, inflame, white, malodorous, smell, open, fluid, serous, and serosanguinous.

\section{Statistical analysis}

Statistical analyses were performed using SPSS ${ }^{\circledR}$ statistical software (v18.0 IBM Corp, New York, NY, USA). Descriptive data were analyzed using frequency distributions. Correlations between variables were calculated using Spearman's rho correlation coefficient. Chi-square analysis with Fisher's exact test was used to examine relationships between various independent variables and the dependent outcome variable of surgical site infection. Odds ratios with $95 \%$ confidence intervals were also calculated. Based on the adult data, factors that were found to be associated with the development of SSI were subsequently entered into a logistic regression model to identify independent predictors of infection. Data are presented as $n(\%)$. Statistical significance was accepted at the $5 \%$ level $(P<0.05)$.

\section{Results}

A total 5023 pediatric patients who underwent surgery from January 2000 to April 2010 were included in the study. The average age of the children in the sample was $4.16 \pm 5.5$ years, and of these, $57 \%$ were boys. Overall, $119(2.37 \%)$ cases of SSI were identified. Table 1 
Table 1 Demographics

\begin{tabular}{lc}
\hline Surgical case type & Cases \\
\hline Cardiac surgery & $3104(61.8)$ \\
Neonate $(\leq 30$ days) & $436(14.0)$ \\
Infant $(>30$ days, $\leq 1$ year) & $827(26.6)$ \\
Child $(>1$ year, $\leq 12$ years) & $1212(39.0)$ \\
Adolescent $(>12$ years, $\leq 18$ years) & $629(20.3)$ \\
ASA $\leq 2$ & $855(27.5)$ \\
ASA $\geq 3$ & $2249(72.5)$ \\
Developed SSI & $72(2.32)$ \\
Spinal/neurosurgery & $338(6.7)$ \\
Neonate $(\leq 30$ days) & $51(15.2)$ \\
Infant $(>30$ days, $\leq 1$ year) & $115(34.0)$ \\
Child $>1$ year, $\leq 12$ years) & $138(40.8)$ \\
Adolescent $(>12$ years, $\leq 18$ years) & $34(10.2$ \\
ASA $\leq 2$ & $91(26.9)$ \\
ASA $\geq 3$ & $247(73.1$ \\
Developed SSI & $8(2.37)$ \\
General surgery & $1581(31.5)$ \\
Neonate $(\leq 30$ days) & $357(22.6)$ \\
Infant $(>30$ days, $\leq 1$ year) & $602(38.1)$ \\
Child $>1$ year, $\leq 12$ years) & $543(34.3)$ \\
Adolescent $(>12$ years, $\leq 18$ years) & $79(5.0)$ \\
ASA $\leq 2$ & $420(26.6)$ \\
ASA $\geq 3$ & $1161(73.4)$ \\
Developed SSI & $39(2.47)$ \\
\hline Data &
\end{tabular}

Data are expressed as $n$ (\%) SSI = surgical site infection, ASA $=$ American Society of Anesthesiologists physical status .

describes the demographics of the sample by case type, American Society of Anesthesiologists physical status, and age and shows that the frequency of SSI was similar regardless of the site of surgery.

Table 2 describes the association between various patient factors and the development of SSIs. As shown, there were no associations between the various patient factors and the development of SSIs although children with diabetes were almost six times more likely to experience an SSI compared with nondiabetic children. However, although this association appears clinically significant, it did not reach statistical significance, likely due to the small number of patients with diabetes. Table 3 describes the effect of antibiotic administration and correct timing on the development of SSI. Children for whom antibiotics were administered incorrectly had a 1.7-fold increased risk of SSIs compared with children who received antibiotics within the recommended guidelines. Interestingly, children who received correctly timed and dosed antibiotics were more likely to suffer an SSI compared with those who did not.

Risk factors included in the logistic regression model were based on prior adult studies $(1-3,5,13,16)$ and included the following: gender, ASA status, tobacco use/exposure, respiratory infection, diabetes, lung
Table 2 SSI factors evaluated

\begin{tabular}{|c|c|c|c|}
\hline Characteristics* & $\begin{array}{l}\text { Non-SSI } \\
N=4904\end{array}$ & $\begin{array}{l}\text { SSI } \\
N=119\end{array}$ & OR (95\% Cl) \\
\hline \multicolumn{4}{|l|}{ Sex } \\
\hline Male & $2798(97.7)$ & $67(2.3)$ & \multirow[t]{2}{*}{$1.03(0.70-1.51)$} \\
\hline Female & $2106(97.7)$ & $52(2.4)$ & \\
\hline \multicolumn{4}{|l|}{ ASA score } \\
\hline$A S A \leq 2$ & $1338(97.5)$ & $34(2.5)$ & \multirow[t]{2}{*}{$0.94(0.62-1.43)$} \\
\hline$A S A \geq 3$ & $3566(97.7)$ & $85(2.3)$ & \\
\hline \multicolumn{4}{|l|}{ ASA emergency } \\
\hline Yes & 492 (97.2) & $14(2.8)$ & \multirow[t]{2}{*}{$1.2(0.65-2.16)$} \\
\hline No & $4412(97.7)$ & $105(2.3)$ & \\
\hline \multicolumn{4}{|l|}{ Tobacco exposure } \\
\hline Yes & $61(98.4)$ & $1(1.6)$ & \multirow[t]{2}{*}{$0.67(0.03-4.54)$} \\
\hline No & 4842 (97.6) & $118(2.4)$ & \\
\hline \multicolumn{4}{|c|}{ Upper respiratory infection } \\
\hline Yes & $102(100)$ & $0(0.0)$ & \multirow[t]{2}{*}{$0.00(0.00-1.93)$} \\
\hline No & $4801(97.6)$ & $119(2.4)$ & \\
\hline \multicolumn{4}{|l|}{ Lung disease } \\
\hline Yes & $486(97.4)$ & $13(2.6)$ & \multirow[t]{2}{*}{$1.12(0.60-2.05)$} \\
\hline No & $4418(97.7)$ & $106(2.3)$ & \\
\hline \multicolumn{4}{|l|}{ Malignancy } \\
\hline Yes & 57 (98.3) & $1(1.7)$ & \multirow[t]{2}{*}{$0.72(0.04-4.87)$} \\
\hline No & $4847(97.6)$ & $118(2.4)$ & \\
\hline \multicolumn{4}{|l|}{ Diabetes } \\
\hline Yes & $7(87.5)$ & $1(12.5)$ & \multirow[t]{2}{*}{$5.92(0.27-48.2)$} \\
\hline No & $4891(97.6)$ & $118(2.4)$ & \\
\hline \multicolumn{4}{|l|}{ Renal disease } \\
\hline Yes & $186(97.9)$ & $4(2.1)$ & \multirow[t]{2}{*}{$0.88(0.27-2.51)$} \\
\hline No & $4718(97.6)$ & $115(2.4)$ & \\
\hline
\end{tabular}

Data are presented as $n(\%), \mathrm{OR}$, odds ratio; $\mathrm{Cl}$, confidence intervals; SSI, surgical site infection.

* Characteristics from history obtained by anesthesia provider and documented in preoperative evaluation.

Table 3 Antibiotic administration

\begin{tabular}{lccc}
\hline & Non-SSI & SSI & OR $(95 \% \mathrm{Cl})$ \\
\hline \multicolumn{2}{l}{ Incorrect } & antibiotic administration** \\
Yes & $814(96.4)$ & $30(3.6)$ & $1.70(1.09,2.63)^{*}$ \\
No & $4090(97.9)$ & $89(2.1)$ & \\
ABX given & & & \\
Yes & $3654(97.1)$ & $108(2.9)$ & $3.36(1.75,6.62)^{*}$ \\
No & $1250(99.1)$ & $11(0.9)$ & \\
\hline
\end{tabular}

$\mathrm{ABX}$, antibiotic; OR, odds ratio; $\mathrm{Cl}$, confidence intervals; $\mathrm{SSI}$, surgical site infection.

${ }^{*} P<0.02$.

**Incorrect antibiotic administration correctly is defined as administering either greater than $1 \mathrm{~h}$ prior to surgical incision, after surgical incision, or administering dose below recommended guidelines.

disease, use of corticosteroids, malignancy, correct antibiotic dose, and time of antibiotic administration. Results identified both incorrect dosing and antibiotic administration as independent risk factors for SSIs in this sample of children. 


\section{Discussion}

This retrospective study was designed to specifically look at the effect of antibiotic prophylaxis in the prevention of SSI in pediatric patients. The overall rate of SSI in our study was $2.37 \%$, which is consistent with the findings of others (4-10). Although studies suggest associations between different factors and the development of SSIs, only a few potential factors can actually be modified to help prevent these infections. According to Bucher et al. (14), postoperative location, urinary catheter insertion, and use of an implantable device are potentially modifiable risk factors for an SSI in children. Results from this study also suggest that the appropriate use of antibiotic prophylaxis is another vital modifiable risk factor and may be the easiest factor to influence. Indeed, our study found that primary failure to administer the correct dose of antibiotics resulted in an almost twofold increase in the risk of developing an SSI (included in incorrect dosing was one case of early administration and one case of late administration of antibiotics). However, children who received an antibiotic had a greater likelihood of developing an SSI compared with children who did not receive antibiotic prophylaxis. The reasons for this are unclear; however, this may simply reflect the fact that the children who did not receive a prophylactic antibiotic were undergoing procedures for which there was no indication and thus were perceived to be at lower risk for SSI. Indeed, the children in our study who did not receive an antibiotic but developed an SSI were older compared with children who did receive a preoperative antibiotic $(8.0 \pm 7.0$ vs $3.5 \pm 5.5$ years) and were likely healthier (ASA IV: $0 \%$ vs $50.9 \%$, respectively) and thus at lower baseline risk. Additionally, we did not break down wound classification, although a majority was class I wound type given the large sample size of cardiac surgery cases. This may also play a predominant role. Additionally, it is possible that some patients did not receive antibiotics because they were already on an appropriate antibiotic regimen and thus prophylactic administration was not warranted.

Recently, emphasis has been placed on the presurgical incision administration of antibiotics to reduce SSI, with reimbursement being tied to proper administration of antibiotics prior to surgery (16). Several recent studies have begun to evaluate Medicare regarding pay for performance measures and outcomes including SSI $(15,17,18)$. Nicholas et al. recently examined, in a retrospective manner, the measures between hospital compliance with surgical care reported on the Hospital Compare website and adverse outcomes. These authors concluded that the lower end compliance hospitals had similar risk-adjusted outcomes on adverse events, including SSI (18). However, questions arise whether reimbursement should be tied to outcomes which have yet to show a correlation with improved outcomes for patients (19). To date, there are few studies that have attempted to look at risk factors for SSI in the pediatric population, more specifically, the role of antibiotic prophylaxis. This is the first study, to our knowledge, to demonstrate the importance of appropriate antibiotic administration in children as a means to reduce SSI. The importance of correct antibiotic usage and dosing plays a major role in decreasing risk of SSI in children.

There are several limitations to our study. First, this study was performed retrospectively at a large, single, tertiary care center. As such, these results may not be generalizable to other institutions whose patient population and medical practice vary. A large population of our sample included cardiac surgery patients and general surgery on neonates leading to high number of patients classified as ASA 4. Second, patients who developed SSI may have been missed if they were discharged home or developed SSI after discharge from hospital and were followed primarily in a clinic or an outside facility. This could lead to an underestimate of actual number of SSI cases. Third, our study did not look at all factors, some of which have been shown to increase the risk of SSI such as placement of indwelling catheters, admission after surgery, placement of an implantable device, and core temperature during surgery due to information availability from our perioperative database. Furthermore, a lack of standardized protocol at our institution regarding choice of antibiotic makes it difficult to access proper decisions. Antibiotic shortages at certain times may have also played a role in altering antibiotic choices. In addition, perhaps patients had an antibiotic allergy preventing use of specific antibiotics, which was not evaluated in this study. Also, we did not breakdown wound classification as a factor. While a majority of cases were class I because of the large sample size of cardiac and spine surgeries, there was a large amount of missing data from general surgery cases, making it difficult to use this as a factor for analysis. Additionally, this study encompassed 10 years, during which the protocol for antibiotic prophylaxis changed to range-based dosing rounded to a common number for children over $2.5 \mathrm{~kg}$ instead of a strict milligram per kilogram dosing. Finally, although the sample size was based on a large convenience sample, we recognize that it may have been insufficiently powered for some analyses.

Results of this study reinforce the importance of correct preoperative antibiotic prophylaxis for the prevention of SSIs in children. Although the results of previous 
studies have been somewhat equivocal, this is the first study to our knowledge showing that failure to provide proper antibiotic prophylaxis increases the risk of SSI in a large group of children. Although there may be several factors that contribute to the development of SSIs, the results of this study suggest that administration of antibiotics in a timely and correct fashion for children is both modifiable and effective. Larger prospective studies are needed to look specifically at timing of antibiotics as well as choice of antibiotics in prevention of SSI as well as other modifiable risk factors.

\section{Acknowledgments}

This research was carried out without funding.

\section{Conflicts of interest}

No conflicts of interest declared.

\section{References}

1 Mangram AJ, Horan TC, Pearson ML et al. Guideline for prevention of surgical site infection, 1999. Hospital Infection Control Practices Advisory Committee. Infect Control Hosp Epidemiol 1999; 20: 250-278.

2 Gagliardi A, Fenech D, Eskicioglu C et al. Factors influencing antibiotic prophylaxis for surgical site infection prevention in general surgery: a review of the literature. Can J Surg 2009; 52: 481-489.

3 Linam WM, Margolis PA, Staat MA et al. Risk factors associated with surgical site infection after pediatric posterior spinal fusion procedure. Infect Control Hosp Epidemiol 2009; 30: 109-116.

4 Doig CM, Wilkinson AW. Wound infection in a children's hospital. Br J Surg 1976; 63: 647-650.

5 Davis SD, Sobocinski K, Hoffmann RG et al. Postoperative wound infections in a children's hospital. Pediatr Infect Dis 1984; 3: 114-116.

6 Sharma LK, Sharma PK. Postoperative wound infection in a pediatric surgical service. J Pediatr Surg 1986; 21: 889-891.

7 Bhattacharyya N, Kosloske AM. Postoperative wound infection in pediatric surgical patients: a study of 676 infants and children. J Pediatr Surg 1990; 25: 125-129.

8 Horwitz JR, Chwals WJ, Doski JJ et al. Pediatric wound infections: a prospective multicenter study. Ann Surg 1998; 227: 553 558.

9 Davenport M, Doig CM. Wound infection in pediatric surgery: a study in 1094 neonates. J Pediatr Surg 1993; 28 : 26-30.

10 Uludag $\mathrm{O}$, Rieu $\mathrm{P}$, Niessen $\mathrm{M}$ et al. Incidence of surgical site infections in pediatric patients: a 3-month prospective study in an academic pediatric surgical unit. Pediatr Surg Int 2000; 16: 417-420.

11 Bratzler DW, Dellinger EP, Olsen KM et al. Clinical practice guidelines for antimicrobial prophylaxis in surgery. Am J Health Syst Pharm 2013; 70: 195-283.

12 Maher KO, VanDerElzen K, Bove EL et al. A retrospective review of three antibiotic prophylaxis regimens for pediatric cardiac surgical patients. Ann Thorac Surg 2002; 74: 1195-1200.

13 Porras-Hernandez JD, Vilar-Compte D, Cashat-Cruz $\mathrm{M}$ et al. A prospective study of surgical site infections in a pediatric hospital in Mexico City. Am J Infect Control 2003; 31: 302-308.

14 Bucher BT, Guth RM, Elward AM et al. Risk factors and outcomes of surgical site infection in children. J Am Coll Surg 2011; 212: 1033-1038 e1031.

15 Hawn MT, Richman JS, Vick CC et al. Timing of surgical antibiotic prophylaxis and the risk of surgical site infection. JAMA Surg 2013; 148: 1-8.

16 Bratzler DW, Houck PM. Antimicrobial prophylaxis for surgery: an advisory statement from the National Surgical Infection Prevention Project. Am J Surg 2005; 189: 395-404.

17 Anthony T, Murray BW, Sum-Ping JT et al. Evaluating an evidence-based bundle for preventing surgical site infection: a randomized trial. Arch Surg 2011; 146: 263-269.

18 Nicholas LH, Osborne NH, Birkmeyer JD et al. Hospital process compliance and surgical outcomes in medicare beneficiaries. Arch Surg 2010; 145: 999-1004.

19 Neumayer L. Timing may not be everything: comment on "Timing of Surgical Antibiotic Prophylaxis and the Risk of Surgical Site Infection". JAMA Surg 2013; 1: 657. 